\title{
Aplicativo T-Learning paraTDT \\ en el Ecuador
}

\section{Resumen}

Este trabajo presenta el desarrollo inicial de una aplicación interactiva T-Learning sobre "Educación Vial". Esta aplicación se rige bajo el estándar de televisión digital ISDB-Tb y fue implementada utilizando el lenguaje Ginga-NCL y el estándar Lua.

Las cifras estadísticas, tomadas de la Agencia Nacional de Tránsito del Ecuador (ANT) desde el 2011 hasta el 2014, muestran que los accidentes de tránsito han incrementado en un $64 \%$ en comparación a los años anteriores. Por este motivo, se vió la oportunidad de difundir a la comunidad sobre temas de seguridad vial a través de un aplicativo T-Learning. Los resultados muestran una gran aceptación por parte de los usuarios en referencia a su facilidad de uso, utilidad y calidad.

\section{Palabras clave:}

Educación, televisión digital, interactiva, T-Learning, ISDB-Tb.

\section{Abstract}

This work presents an initial development of an interactive T-Learning application about "Traffic Education". The application is regulated under the digital television standard ISDB-Tb and it was implemented using Ginga-NCL programming language and Lua standard.

Statistics taken from Ecuadorian Transit Regulation Organism (ANT) had shown that road traffic accidents increased 64\% from 2011 to 2014 compared to previous years. Therefore, traffic education was recognized as a social issue that people could learn by using the T-Learning application. Results showed a good acceptance by users while using the application in terms of ease of use, usefulness and product quality.

\section{Keywords:}

Education, digital tv, interactivity, T-Learning, ISDB-Tb. 


\section{Introducción}

Para desarrollar un prototipo de aplicación interactiva T-Learning para la "Educación Vial" es necesario que previamente se considere, como circunstancia vinculante, que en el Ecuador hay un apreciable porcentaje de la población que ha desertado en los diferentes niveles de formación educativa menores que los que se encuentran para América Latina (Unicef, $s / f)$.

De acuerdo a indicadores obtenidos, por la Institución Contrato Social por la Educación Ecuador, en el periodo 2009-2010, el $3,90 \%$ de la población ecuatoriana desertó en los niveles de educación básica; sin embargo, en el 2011-2012, esta cifra se incrementó a $4,80 \%$. Por otro lado, en el bachillerato se obtuvo el 5,50\% de deserción en el periodo 2009-2010 cifra que se incrementó al 5,90\% en el 2011-2012 (Contrato Social por la Educacion Ecuador, s/f).

Las necesidades de la población se extienden más allá de la deserción en niveles de educación formal, pues se requiere complementar la educación formal e informal, con el propósito de mejorar la capacidad de entendimiento y de discernimiento.

El objetivo general de este trabajo es proponer aplicaciones T-Learning como una herramienta para la difusión de programas de capacitación y formación, en todos los ámbitos del conocimiento, que existen o que se puedan crear en el país, proponiendo una estructura modelo para el desarrollo de aplicaciones educativas para la televisión digital.

El objetivo específico es proponer un modelo de aplicación educativa relacionada con la educación vial, utilizando la televisión digital. A partir de los resultados de orden particular que se obtenga y utilizando el método deductivo, se llegará al objetivo general.

Para elaborar esta propuesta se analizaron proyectos que existen en otros países; así como estadísticas relacionadas con la educación, acceso a la televisión y tecnologías disponibles que nos permitieron visualizar mecanismos de convergencia para construir un aplicativo T-Learning.

Con la entrada en vigencia de esta propuesta se pretende suplir las eventuales deficiencias ocasionadas por la deserción, de un apreciable porcentaje de la población educativa, en los niveles de instrucción primaria y/o secundaria; y complementar el nivel de educación que se propone en actuales programas de capacitación desarrollados por instituciones como: el SECAP, que es una dependencia del Ministerio de Relaciones Laborales. Aprendamos, que es un programa de formación ofrecido por Fundación Ecuador y el Municipio de Guayaquil y Educa, que es un programa de formación del gobierno ecuatoriano manejado por el Ministerio de Educación MinEduc, entre otros.

\section{Marco referencial de la Televisión Digital}

En el año 2003 la Fundación Ecuador, con el auspicio del Municipio de Guayaquil, ofreció por televisión una serie de cursos educativos de utilidad variada, en un programa llamado "Aprendamos" que se sintoniza a nivel nacional en diferentes horarios y en el que se han registrado oficialmente 984.837 personas (Fundación Ecuador, 2010).

Posteriormente, el gobierno ecuatoriano ofreció una serie de cursos con el membrete de un programa llamado "Educa" que funciona con una metodología diferente a la de "Aprendamos" (Educa, s/f).

Sin embargo del esfuerzo desarrollado, ambos programas de televisión están sujetos a horarios rígidos y obedecen exclusivamente a material de contenido didáctico, por lo que los resultados no son los esperados.

A pesar de lo anterior, se reconoce que la televisión es uno de los medios de comuni- 
cación que mayor influencia ejerce en la sociedad debido a que, por su ingreso a los hogares, impacta de manera directa a los televidentes informando, entreteniendo $y$ educando (Zajc, Alič, Battelino, \& Tasič, 2007).

Por su gran trascendencia este medio se encuentra en constante evolución, tal como ocurre en la actualidad, cuando somos testigos de uno de los grandes cambios en la televisión, como es la migración de la tecnología analógica a la digital.

En el año 2010 el gobierno ecuatoriano, mediante una resolución del Consejo Nacional de Telecomunicaciones CONATEL, aprobó la migración de la señal de televisión analógica a digital, proceso que se espera que culmine el año 2017 (Ministerio de Telecomunicaciones y de la Sociedad de la Información, s/f).

La televisión digital TD forma parte de una nueva generación en el proceso de evolución de la tecnología de transmisión-recepción de las señales de comunicación (dos Santos, do Vale, \& Pedroso, 2006), lo que representa una mejora a gran escala en la señal televisiva y permite optimizar los recursos del espectro radioeléctrico logrando incrementar la calidad de la imagen, del sonido $y$, permitiendo la accesibilidad a múltiples canales y servicios interactivos (Pindado, 2006), causando con ello un gran impacto en el desarrollo social y un gran beneficio al usuario final.

Esta innovación trae consigo una extensa lista de servicios en los diferentes campos del desarrollo como son: el comercio, la gestión administrativa, la educación, la salud, el entretenimiento, etc. A las actividades antes mencionadas, se las conoce como T-Comercio, T-Gobierno, T-Learning, T-Salud y T-Entretenimiento respectivamente. Cabe añadir que esta tecnología también presta servicios actualmente asociados a un computador, como lo son el acceso a Internet y correo electrónico (Reyes, Moreno, \& Rose- ro, 2010).

La iniciativa de este cambio de tecnología ha sido acogida por la mayoría de los gobiernos en el mundo, para implementar la Televisión Digital Terrestre TDT. Esto ha permitido una elevada incursión y ha dado a conocer las ventajas de la tecnología digital en la televisión. Adicionalmente, se ha reconocido que el televisor se podría convertir en un medio de uso cotidiano y familiar para investigar $e$ incursionar en nuevas áreas de desarrollo que generen una mayor inclusión social (Reyes \& Moreno, 2009).

El T-Learning se prevé como una alternativa de educación gratuita, eficiente, con mayor penetración y alcance en todo el país. De acuerdo con la Encuesta Nacional de Empleo Desempleo y Subempleo (ENEMDUR) del INEC, el $86.4 \%$ de los ecuatorianos cuentan con televisores en sus hogares y en promedio dedican seis horas al día a ver televisión (Instituto Nacional de Estadísticas y Censos INEC, s/f).

De manera general, la televisión digital facilita una comunicación bilateral interactiva entre el televidente y el proveedor del contenido (Lytras, Lougos, P., \& Pouloudi, 2002). El tener dicho canal de retorno, proporciona la convergencia con las diferentes tecnologías web, permitiendo obtener y enviar contenidos a través del internet por medio de la televisión (dos Santos, do Vale, \& Pedroso, 2006).

Adicionalmente, la televisión digital aporta grandes ventajas sobre la televisión analógica, entre las cuales tenemos: mayor cantidad de canales en un mismo ancho de banda, nuevos contenidos y servicios interactivos, mejor recepción y transmisión de la señal, recepción portátil y en movimiento; y mejor calidad de servicio a nivel de imagen y sonido (Reyes \& Moreno, 2009).

Específicamente, la televisión digital re- 
copila tres beneficios generales a nivel de usuario que son (Jiménez \& Moreno, 2012): interactividad, que implica que el usuario final puede controlar las actividades y sus acciones; personalización, para el contenido interactivo que se adapta a cada perfil de usuario y digitalización, para satisfacción y confort del usuario debido a la alta calidad de audio y video.

La interactividad es el beneficio clave de la televisión digital y es el que lo convierte en una herramienta de alto impacto para el televidente.

\section{Interacción e Intercatividad}

La Televisión Digital (TD) es una tecnología que puede influenciar fácilmente, si es utilizada de manera apropiada, debido al elevado índice de penetración que tiene la televisión al ser repotenciada con la variable interactividad. La unión de ambas se la conoce con la denominación de Televisión Digital interactiva (TDi).

La interactividad permite cambiar el escenario estático del televidente a uno totalmente dinámico, añadiendo la posibilidad de personalizar diferentes tipos de servicios que pueden ser ofrecidos por este medio (Acevedo, Arciniegas, García, \& Perrinet, 2010).

En otras palabras, la interactividad es la capacidad que tiene el televidente para personalizar el contenido televisivo, de acuerdo a las opciones que disponga. Su interacción es individualizada gracias a la existencia del componente clave que es el canal de retorno, que permite la interactividad y la diferencia de las transmisiones masivas. Esta característica comunicacional crea una relación televidente/televisor, logra darle al usuario la potestad de controlar libremente el contenido presentado y una agradable percepción de usuario con experiencia en tecnologías de la información (Pindado, 2006).
En cuanto al uso del canal de retorno, se definen tres niveles de interactividad en la TDi que son: interactividad local, cuando el usuario no tiene acceso al canal de retorno; interactividad parcial, cuando el usuario no tiene acceso al canal de retorno en ciertos momentos del día; e, interactividad completa, cuando el usuario tiene acceso ininterrumpido al canal de retorno con contenido de tiempo real. En términos generales, la TDi es la convergencia de la televisión con las computadoras que tienen acceso a internet. Esto es mediante la personalización y heterogeneidad de contenidos que brinda la TD, permitiendo así, considerar la migración de sistemas de E-Learning a la televisión (dos Santos, do Vale, \& Pedroso, 2006).

No obstante, para que se pueda contar con el canal de retorno que permite la interactividad en el Ecuador, los televisores deben contar con los llamados set-top-box en el caso de que sean antiguos o que cuenten con una norma diferente de fábrica. Este es un codificador que transforma la señal de tecnología digital a análoga permitiendo la recepción de canales digitales y aplicaciones interactivas. De esta manera se logra brindar al usuario todos los beneficios de la televisión digital sin importar el modelo del televisor. Adicionalmente, gracias al set-top-box, el receptor de televisión se convierte en un terminal multimedia, que tiene la capacidad de tener las mismas funcionalidades de interacción en línea que un computador con acceso a internet. El set-top-box ha establecido al televisor como una mejor alternativa al uso de computadoras y tabletas (Dávila, 2012); por lo que sería necesario que se implemente una política de estado que permita adquirir el set-top-box a la población de escasos recursos.

Con la llegada de la TDi muchas áreas de desarrollo se posicionan los diferentes tipos de aplicaciones para proveer servicios de calidad a los usuarios. Para este caso específico tenemos a las aplicaciones de tipo T-Lear- 
ning. Como se mencionó anteriormente, esta área se enfoca en el área educativa, como una alternativa viable de desarrollo social. De este modo entra en comparación y contraste con una tecnología existente, E-Learning. Para poder identificar sus similitudes y diferencias vamos a definir cada una.

E-Learning, en términos generales, es el uso de la tecnología para fines educativos; es por esto que se lo considera más una tendencia social que tecnológica, ya que el fin principal es la enseñanza, independientemente del medio a utilizar. Para lograr este objetivo, enfoca su atención en el desarrollo de procesos eficientes y flexibles. De esta manera, garantiza que el contenido llegue a la mayoría de estudiantes y profesores (Lytras, Lougos, P., \& Pouloudi, 2002).

$\mathrm{Al}$ tener un constante y mejor acceso a la información en la internet, se ha conseguido mejorar los ambientes de aprendizaje convirtiéndolos en estructuras más dinámicas e interactivas. Esto se debe a que los diferentes entornos de recreación y trabajo tienen disponibles herramientas que ayudan tanto al maestro como al estudiante a interactuar, acceder y compartir conocimiento (Ling-Lang, Che-Han, \& K., 2014).

Es por esto que, los sistemas de e-learning han logrado evolucionar de ambientes estáticos a sistemas con contenido personalizado, de acuerdo al tipo de usuario, ya que cada estudiante, demanda muchas veces a que el software se adapte utilizando técnicas diferentes de acercamiento garantizando la efectividad de una educación a distancia (Alshammari, Anane, \& Hendley, 2014).

Se conoce al T-Learning como el proceso de enseñanza y aprendizaje mediante el uso de la TDi. Específicamente, es la convergencia de la tecnología televisiva, las telecomunicaciones y los sistemas informáticos en conjunto con el sector educativo; y, la interacción audiovisual (Reyes, Moreno, \& Rosero, 2010)

En la figura 1 se muestra al T-Learning como la convergencia del E-learning, el computador y el televisor.

Figura 1.

Diagrama de convergencia de E-Learning, Televisión y Computador.

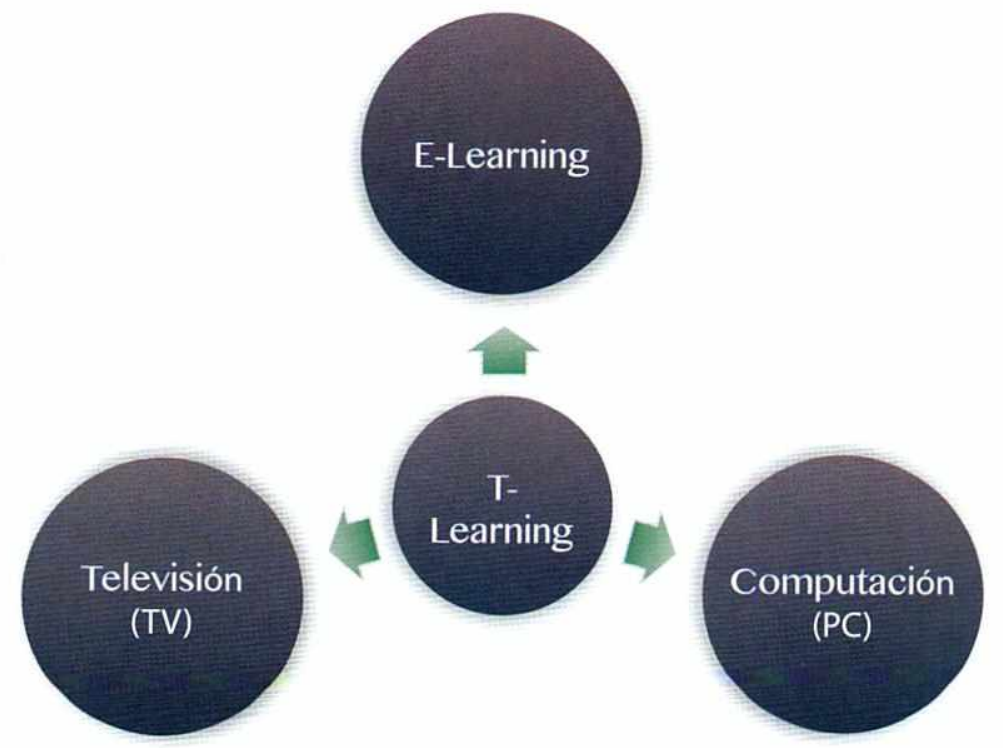

Fuente: Adaptado de Jiménez, Moreno (2012). Cycle of PDCA T-Learning model and its application on Interactive Digital TV. 
Por otro lado, T-Learning incluye otras áreas de desarrollo mediante el uso de tecnologías móviles y protocolos de internet. Sin embargo, la televisión o cualquier otro dispositivo configurable para ver contenido televisivo es el principal medio en esta modalidad de aprendizaje, mientras que el resto de dispositivos son detallados como medios secundarios para el aprendizaje (Reyes \& Moreno, 2009).

Las aplicaciones de T-Learning cuentan con una subclasificación de acuerdo al enfoque. Esto va en relación a la formalidad del tipo de aprendizaje. En esta división encontramos cursos de aprendizaje formal e informal, material de soporte a los cursos de aprendizaje formales dados en instituciones educativas, cursos de entrenamiento especial, elementos de soporte en cuidado del hogar, cursos personalizados profesionales, módulos de carácter social y de soporte, jornadas de entrenamiento para inmigrantes para el proceso de adaptación, programas de entrenamiento para personas que se encuentran en áreas remotas, entre otros (Jiménez \& Moreno, 2012).

También es importante considerar que del otro lado del televisor nos encontramos con televidentes que tienen diferentes expectativas de un programa. Estas personas pueden estar consciente o inconscientemente utilizando el aplicativo T-Learning.

Es por esto que, cuando se realicen mediciones o se tomen estadísticas, se debe tomar a consideración que nos podemos topar con diferentes tipos de usuarios. Estos grupos de usuarios son personas que están buscando programas educativos, que no tienen o no utilizan un ordenador, personas muy jóvenes o muy avanzadas de edad para usar un ordenador, que no cuentan con la capacidad económica para un computador, personas con falta de motivación para aprender en en este medio, que no tienen acceso a internet $\mathrm{u}$ otros (Reyes, Moreno, \& Rosero, 2010).
En resumen, es de vital importancia conocer los tipos de televidentes en un ciclo de vida de un aplicativo T-Learning. De esta manera se puede definir el tipo de usuario al que se quiere llegar y de qué manera se debe adaptar. Por lo general se debe poner énfasis en los siguientes atributos para los perfiles: información de la familia, perfil educativo, herramientas tecnológicas disponibles, patrones del televidente que son los horarios y programas vistos, Etc. (Zajc, Alič, Battelino, \& Tasič, 2007).

\section{Casos de Estudio T-Learning}

La implementación de sistemas T-Learning es una iniciativa que la han tomado diferentes países desde hace aproximadamente once años con resultados altamente positivos, logrando romper paradigmas en cuanto a la televisión como un medio de distracción y no de aprendizaje. En el año 2002, la cadena de televisión BBC introdujo un programa T-Learning llamado "Caminando entre las bestias" (Walking With Beasts), que permitía ver el detalle de cada episodio, especificidades de cada dinosaurio, información adicional y creación del episodio. Los usuarios, que contaban con el decodificador apropiado, pudieron interactuar con el programa que tuvo gran acogida (Pindado, 2006).

Por otro lado, en el año 2005, se desarrolló una aplicación T-Learning en Finlandia para niños entre 9 y 11 años de gran desarrollo que consistió en complementar el contenido recibido en la escuela desde sus hogares. Esta aplicación les permitía interactuar entre ellos. Siguiendo este ejemplo, se desarrollaron otro tipo de servicios interactivos dirigidos a otros grupos como personas mayores y discapacitados (AARRENIEMI-JOKIPELTO, 2005).

Este movimiento se trasladó a Italia, que a partir del año 2006, optó por entrar a la TDi con gran intensidad. Estos países, son los que más cuentan con una variedad de servicios interactivos además de T-Learn- 
ing. El programa que mayor acogida tuvo a nivel nacional se llamó Television Language, un programa interactivo de enseñanza de idiomas con módulos de evaluación (Nicolussi, 2005).

En el 2007 en España, la Universidad de Vigo y CESGA, crearon un aplicativo T-Learning conocido como T-Maestro. Este aplicativo basa su desarrollo en dispositivos de recepción de televisión digital (televisores y dispositivos móviles) para traer como producto final, aplicaciones telemáticas interactivas. El proyecto finalizó en el año 2010 (CESGA, 2007).

En el año 2007, la Unión Europea y Brasil iniciaron en conjunto el proyecto Beacon. Este proyecto tuvo tres objetivos: interoperabilidad entre Brasil y la UE en relación a estándares de televisión digital, el estudio de una metodología para educación a distancia mediante la televisión digital, y la entrega de servicios de tipo T-Learning relacionados con la inclusión social en Sao Paulo, Brasil. El proyecto finalizó en el año 2010 (BEACON, 2007).

Tras consolidarse este tipo de servicios mediante el uso de la TD, un grupo de investigadores en una conferencia en China en el año 2008, propusieron un modelo para aplicaciones T-Learning enfocándose en dispositivos móviles. El trabajo estaba motivado por la falta de continuidad del televidente debido a las limitaciones tecnológicas existentes en el medio y el desarrollo apropiado de aplicaciones T-Learning para cualquier dispositivo (Chao-Chun, Yong-Ming, Lien-Fa, \& Ding-Chau, 2008).

Dada la gran tendencia de implementar aplicaciones para TD, un grupo de investigadores elaboró un marco de trabajo en el año 2010, para estandarizar las aplicaciones T-Learning, que define parámetros a seguir y aspectos a considerar en el desarrollo de este tipo de aplicaciones (Bellotti, et al., 2010).

Los casos de estudio anteriormente mencionados proveen suficiente soporte para la TDi, ya que todo lo que se busca es informar, educar y entretener al usuario a través de herramientas alternas; tal es el caso de la televisión con el uso de componentes dinámicos. Estas son las aplicaciones que permiten al televidente interactuar de manera eficiente.

\section{Metodología}

En la figura 2 se presenta un diagrama de bloques que contiene en síntesis la metodología que se ha seguido para desarrollar el modelo de aplicación T-Learning.

\section{Figura 2.}

Diagrama de bloques del aplicativo T-Learning desarrollado

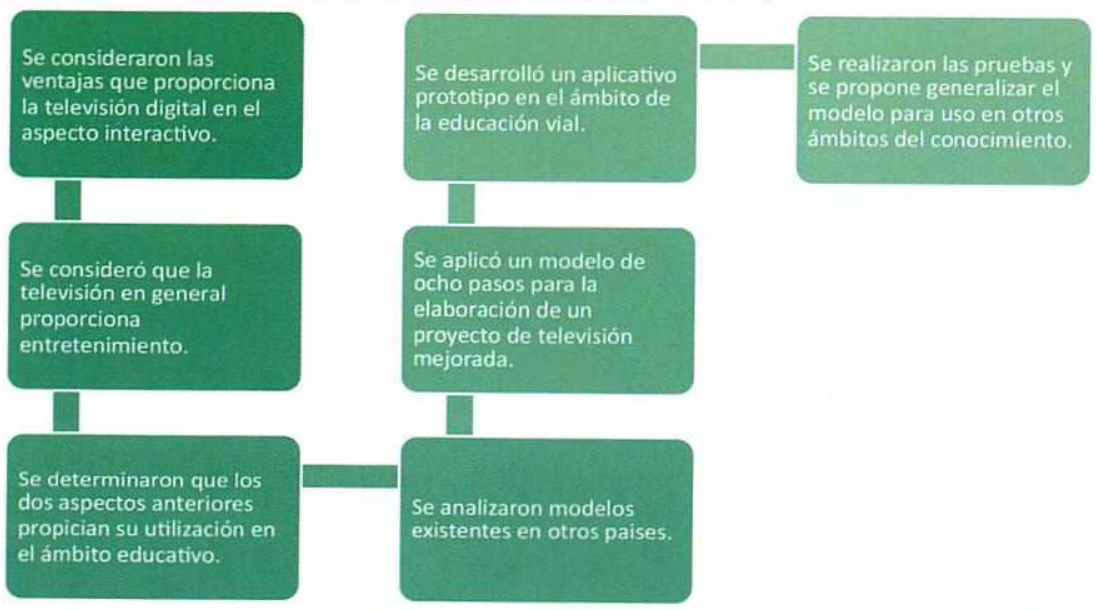


Para el desarrollo de la aplicación T-Learning se utilizó el lenguaje Ginga-NCL con Lua, que se asumió tomando como base la explicación de lenguajes declarativos y procedimentales de Sandoval (Sandoval, 2011), por su alto nivel de abstracción y descripción de tareas; $y$, por que las aplicaciones en Ginga-NCL, son más fáciles de concebir y entender.

Ginga es un middleware de código abierto utilizado exclusivamente para el estándar ISDB-Tb, que permite la ejecución del lenguaje declarativo Nested Context Language NCL y Lua (Villanueva \& Velásquez, 2010), su arquitectura se encuentra dividida en módulos basados en la programación y el uso del middleware (Comunidad Ginga Ecuador, s/f). NCL es un lenguaje desarrollado utilizando una estructura modular basada en la web, lo que permite una alta escalabilidad en las aplicaciones desarrolladas. Este lenguaje es netamente declarativo; por lo que basa su funcionamiento en la construcción de documentos hipermedia (Nested Context Language, s/f).

En cuanto a la codificación, la International Telecommunication Union (2011), establece que el diseño de un documento NCL debe estar estructurado por: un encabezado que contiene la descripción del documento y la definición de descriptores, conectores y regiones; y por un cuerpo que es la sección donde se definen los contextos, los nodos y los enlaces entre los mismos.

Lua es un lenguaje de scripting desarrollado e implementado por la PUC-Río que realiza programación imperativa, es decir, se basa en términos del estado del programa y sentencias para modificarlo. Esta es una plataforma liviana de scripting, debido a que su código fuente es de $860 \mathrm{~Kb}$ que permite utilizarlo en aplicaciones electrónicas con limitaciones de memoria (Ierusalimschy, de Figueiredo, \& Celes, 2006).
Para el desarrollo de la metodología usada se analizaron algunos casos que determinaron los aspectos positivos de cada uno de ellos, con el propósito de integrarlos a la lógica del aplicativo y mejorar de esta manera la experiencia del usuario. Al aplicar estos cambios se logró estandarizar el desarrollo del código, las interfaces de la solución y la adición de funcionalidades como primer paso para adoptar el marco de trabajo propuesto por Belloti (Bellotti, et al., 2010).

Uno de los casos que se tomó en consideración fue el proceso de 8 pasos para la construcción de televisión mejorada convincente de Microsoft (Lamont, 2003), el cual determinó el enfoque y el orden para la implementación del aplicativo T-Learning.

Otro caso fue el del estudio del caso de Finlandia (AARRENIEMI-JOKIPELTO, 2005); se tomó como base el aplicativo de la interacción televidente-televisor, debido a que se enfoca en fortalecer la lógica del contenido que se presenta.

Con relación al aplicativo T-Learning de la BBC (Pindado, 2006), "Caminando Entre las Bestias", se consideró incluir una opción de resumen de cada episodio, con la condición de que el contenido transmitido no se pierda de vista mientras se lee el resumen y para que el usuario pueda observar el episodio de forma simultánea al contenido del mismo.

También se consideraron las observaciones realizadas en el trabajo presentado en la conferencia de Kaohsiung (Chao-Chun, Yong-Ming, Lien-Fa, \& Ding-Chau, 2008), pero no fueron aplicadas debido a que en el Ecuador no se encuentra implementado el estándar de televisión digital, en su totalidad.

El área de enfoque escogida para el desarrollo de la aplicación T-Learning 
prototipo fue la educación vial, debido al elevado grado de relevancia que establece la ONU (Organización de Naciones Unidas ONU, s/f) para la seguridad vial.

Los recursos para la construcción de este prototipo fueron obtenidos del sitio web de la Agencia Nacional de Transito ANT, mientras que los videos fueron obtenidos a través de una búsqueda en internet. Toda esta información es de libre acceso y gratuita.

El prototipo T-Learning consta de tres módulos con: señales reglamentarias, señales de advertencia y señales informativas. Cada uno de estos módulos consta de un video que tiene una duración de aproximadamente cinco minutos y durante su reproducción el usuario puede desplegar un menú que tiene tres opciones: contenido del episodio, información adicional complementaria y evaluación.

Al seleccionar la opción de contenido del episodio, se reduce el tamaño del video y se muestra un pequeño resumen del mismo; cuando se escoge la opción información adicional complementaria, muestra un contenido extra, relevante al episodio. Finalmente, cuando se escoge la opción evaluación, esta lleva al usuario a rendir una prueba de cinco preguntas, de opción múltiple, sobre el contenido revisado. Una vez completada la evaluación, el usuario obtendrá una calificación que comprobará su grado de entendimiento respecto al tema tratado en el episodio.

En razón de que no se cuenta con la infraestructura adecuada para la ejecución de la aplicación diseñada, en reemplazo de un televisor con el servicio de señal digital, se usó una computadora personal que cuenta con un programa que emula la aplicación.

Para acceder al servicio, el usuario debe desplegar secuencialmente las cuatro opciones que están identificadas por los cuatro botones de interacción del control remoto, los mismos que son simulados por caracteres del teclado que se observan en la figura 3 y que se detallan a continuación:

- Tecla "F1": Botón de interacción rojo del control remoto.

- Tecla "F2": Botón de interacción azul del control remoto.

- Tecla "F3": Botón de interacción verde del control remoto.

- Tecla "F4": Botón de interacción amarillo del control remoto.

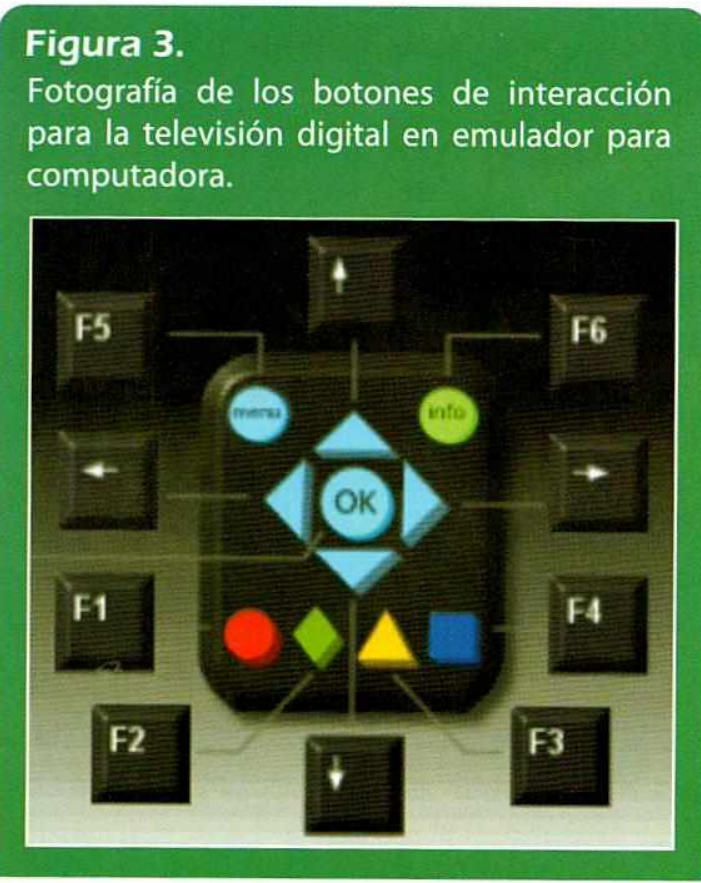

El botón rojo despliega el menú inicial. A partir de este momento las opciones que el usuario puede escoger se identifican con el color de cada botón, los mismos que se ejecutan después del comando del usuario.
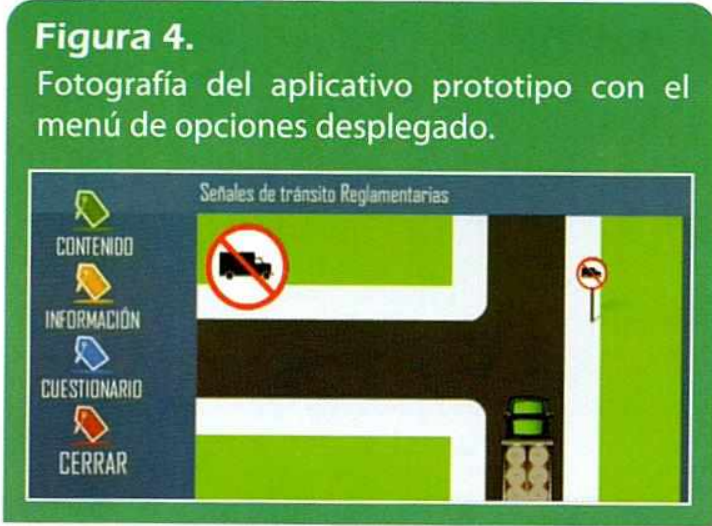


\section{Análisis de Resultados}

Con el objetivo de evaluar la eficiencia y eficacia del aplicativo T-Learning, se pidió a un grupo de diez personas que hagan uso de la herramienta desarrollada, para tener resultados objetivos. Las pruebas se realizaron con dos personas de cada uno de los cinco grupos, comprendidos entre las edades definidas por la Encuesta Nacional de Empleo Desempleo y Subempleo (ENEMDUR) del INEC que probaron e interactuaron con el aplicativo, mediante la sintonización del contenido mostrado en el televisor.

Para evaluar el rendimiento de la aplicación se definieron cuatro variables, en las que a cada usuario se le pidió que calificara del uno al diez, siendo diez el máximo puntaje.

\section{Las variables son:}

1. Facilidad de manejo del aplicativo: el objetivo de esta variable es calificar el nivel de dificultad en el manejo de la aplicación.

2. Utilidad del contenido presentado: el objetivo de esta variable es determinar que las diferentes opciones brindadas en el aplicativo sirvan para enriquecer cultural y educativamente al usuario.

3. Calidad del prototipo: está determinado por el grado de comprensión que genere en el usuario la información suminstrada.

4. Ventajas del prototipo: es un modelo entretenido, educativo y formativo, al que se accede por voluntad y decisión del usuario.

Una vez que los usuarios emitieron su calificación se calculó un promedio de cada una de estas variables por grupo de edad. Sus resultados se pueden observar en la figura 5 .

\section{Figura 5.}

Gráfico con las calificaciones del prototipo de aplicativo T-Learning por grupos de usuarios locales.

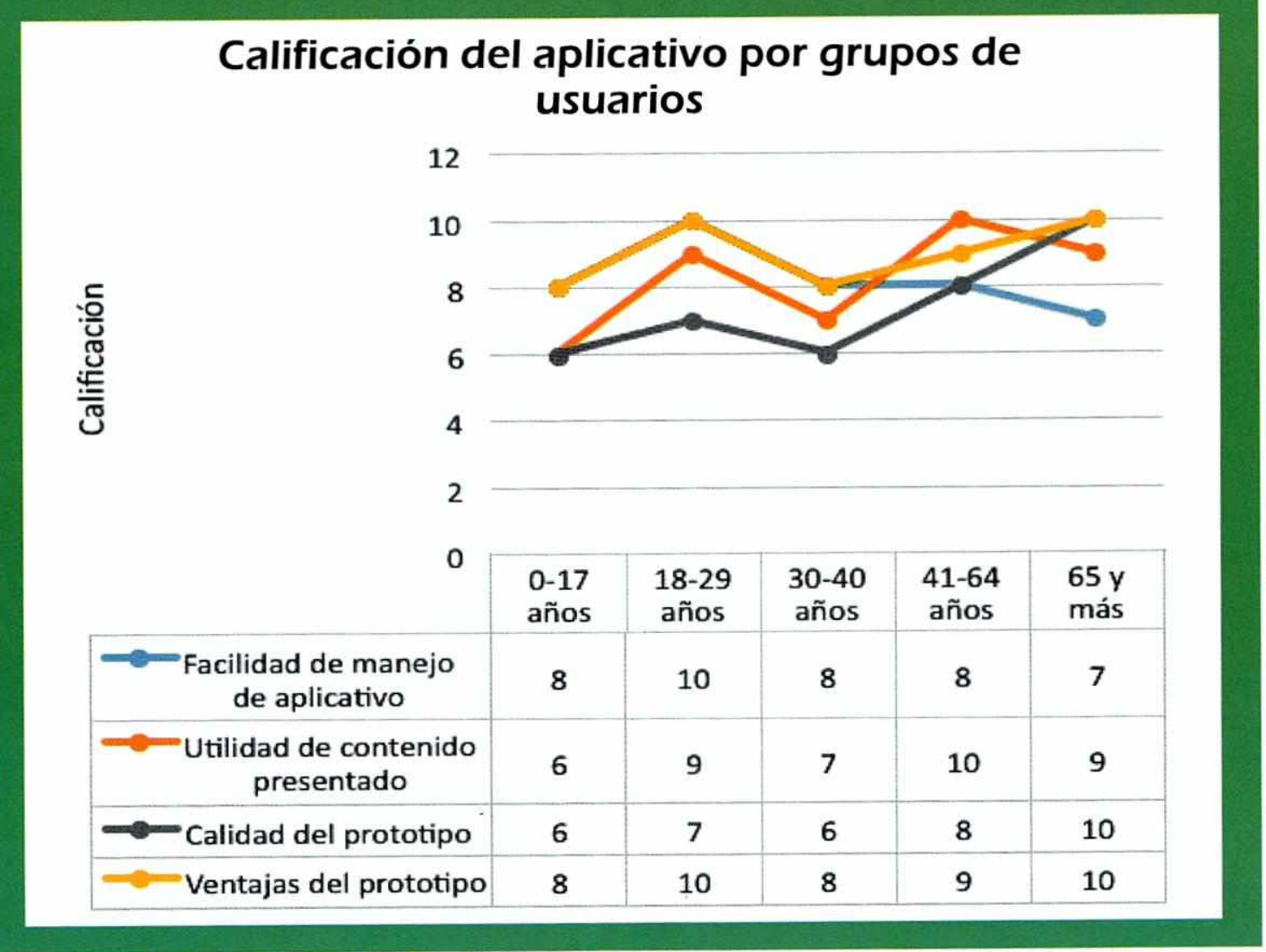


Estas valoraciones son referenciales, pues han sido realizadas por muestreo y sobre una pequeña masa de la población; por lo tanto pueden variar en función de la magnitud de la muestra y de la complejidad del tema difundir vía T-Learning.

El grupo comprendido entre 18-29 años de edad, fue el que encontró más facil de manejar el aplicativo, mientras que más difícil se le hizo al grupo de 65 años y más.

El grupo comprendido entre 41-64 años de edad, fue el que encontró más útil el contenido presentado; mientras que el grupo de 0-17 años encontró menor utilidad en el contenido presentado. En la masa poblacional comprendida hasta los 17 años, por su nivel de adaptabilidad a las nuevas tecnologías de la información, es más exigente en cuanto a la calidad del producto, mientras que los mayores a 65 años privilegian la facilidad del acceso a la calidad del producto. En cuanto a las ventajas hay una apreciación generalizada sobre la conveniencia y ventajas que representa el prototipo.

Con los resultados obtenidos, no sólamente se determina la calidad del trabajo realizado; sino que también se establece ¿en qué variables el usuario tiene mayor nivel de exigencia, parámetros con los que se genera una tendencia de mejora constante para la aplicación.

\section{Conclusiones y Recomendaciones}

El programa que se genere para estas aplicaciones requiere de un equipo especializado que desarrolle el contenido televisivo.

Los usuarios correspondientes a los diferentes rangos de edad se adaptaron con facilidad a los comandos de operación del prototipo, debido a la familiarización que tienen con el uso del televisor y de su control remoto. Sin embargo, es necesario explicar en detalle el manejo de la aplicación y sus comandos de interacción.
El componente de interactividad jugó un importante rol en las pruebas, debido a que los usuarios pudieron navegar ininterrumpidamente a través del aplicativo, mientras seguían sintonizando el programa.

Los usuarios que contribuyeron con la encuesta realizada, manejaron el prototipo de aplicación, lo encontraron interesante y altamente educativo, motivo por el cual las preguntas presentadas en las evaluaciones, constan en el examen que se rinde para la obtención y renovación de licencias de conducir.

En general, la TDT y la TDi, proponen una vía de aprendizaje diferente, de mayor alcance y de alta penetración con aplicaciones de tipo T-Learning, que no solamente es gratuita si no que tiene más acogida fundamentada en la popularidad del uso de la televisión.

Una vez que se implemente la infraestructura de televisión digital en el país, los primeros programas de eduación vial deben ser dirigidos hacia los grupos humanos, comprendidos hasta los 64 años de edad; de acuerdo con los resultados mostrados en este documento, puesto que son los que mayor facilidad encuentran en el manejo del aplicativo.

Se debe conformar una alianza estratégica entre las estaciones de televisión pública y privada, que alcancen la migración a la televisión digital, con las instituciones educativas formales y no formales, para emprender un plan maestro masivo de capacitación de la sociedad ecuatoriana.

\section{Glosario}

\section{Interactividad}

La interactividad es la capacidad que tiene el televidente para personalizar el contenido televisivo (Acevedo, Arciniegas, García, \& Perrinet, 2010). 


\section{Televisión Analógica}

Es la radiodifusión, de una sola vía, de programación televisiva destinada a ser sintonizada por los televidentes en general a través de una estación de difusión (Agencia Nacional de Televisión de Colombia, (s/f)).

\section{Televisión Digital}

Es una tecnología de transmisión de señal, en donde las señales que se envían y se reciben pasan a un formato digital. Esto representa una mejora a gran escala en recepción de la señal televisiva y permite optimizar los recursos del espectro radioeléctrico (dos Santos, do Vale, \& Pedroso, 2006).

\section{T-Learning}

Es el proceso de enseñanza y aprendizaje mediante el uso de la TDi. También busca converger la tecnología televisiva, telecomunicaciones y sistemas informáticos con el sector educativo y la interacción audiovisual (Reyes \& Moreno, 2009).

\section{E-Learning}

Es el uso de la tecnología para fines educativos. Se lo considera más una tendencia social que tecnológica, ya que el objetivo principal del E-Learning es garantizar un aprendizaje efectivo (Lytras, Lougos, P., \& Pouloudi, 2002).

\section{ISDB-TB}

Es la norma de televisión digital construida por Japón y modificada por Brasil. Es el estándar que se escogió para el Ecuador (Dávila, 2012).

\section{GINGA}

Ginga es un middleware de código abierto utilizado exclusivamente para el estándar ISDB-Tb. El mismo permite la ejecución del lenguaje declarativo Nested Context Language NCL y Lua (Comunidad Ginga Brasil, s/f).

\section{NCL}

Es un lenguaje desarrollado utilizando una estructura modular basada en la web, lo que permite una alta escalabilidad en las aplicaciones desarrolladas. Este lenguaje es netamente declarativo, por lo que basa su funcionamiento en la construcción de documentos hipermedia (Nested Context Language, $\mathrm{s} / \mathrm{f}$ ).

\section{LUA}

Lua es un lenguaje de scripting, desarrollado e implementado por la PUC-Río que realiza programación imperativa, es decir, se basa en términos del estado de programa y sentencias para modificarlo (Pontificia Universidad Católica de Río de Janeiro, s/f).

\section{SET-TOP-BOX}

Es un codificador que se conecta al televisor. Transforma la señal de tecnología digital a análoga permitiendo la recepción de canales digitales y aplicaciones interactivas (Dávila, 2012).

\section{MIDDLEWARE}

Un middleware es un software que permite la interacción entre aplicaciones, redes, software y hardware (Villanueva \& Velásquez, 2010).

\section{Referencias Bibliográficas}

AARRENIEMI-JOKIPELTO, P. (2005). $T$-learning Model for Learning via Digital TV. Proceedings of the 16th EAEEIE AnnualConference on Innovation in Education for Electrical andInformation Engineering (EIE).

Acevedo, C., Arciniegas, J., García, X., \& Perrinet, J. (2010). Proceso de Adaptación de una Aplicación de e-aprendizaje a t-aprendizaje. Congreso de Educación.

Agencia Nacional de Televisión de Colombia. ((s/f)). From http://www.antv.gov.co/atencion-a-usuarios/preguntas-frecuentes/television-abierta/que-es-la-television-analoga-terrestre 
Alshammari, M., Anane, R., \& Hendley, R. (2014). Adaptivity in E-Learning Systems. Complex, Intelligent and Software Intensive Systems (CISIS) , 79-86.

BEACON. (2007). From http://www.abed.org.br/congresso2009/cd/trabalhos/2462009180741.pdf

Bellotti, F., Vrochidis, S., Pelegrino, M., Tsampoulatidis, I., Lhoas, P., Bo, G., et al. (2010). An integrated framework for personalized t-learning. IGI GLOBAL.

CESGA. (2007). From https://www.cesga.es/es/investigacion/proyectos/Proyecto? $\mathrm{id}=159$

Chao-Chun, C., Yong-Ming, H., Lien-Fa, L., \& Ding-Chau, W. (2008). Genetic Optimization for Benefit-Oriented Data Broadcast in T-Learning Environments. Intelligent Systems Design and Applications, (pp. 403-408).

Comunidad Ginga Brasil. (s/f). From http://www.ginga.org.br/

Comunidad Ginga Ecuador. (s/f). From http://www.ginga.org.ed/

Contrato Social por la Educacion Ecuador. (s/f). Educacion en Cifras. Retrieved 16 de julio de 2013 from http://www.educacionencifras.ec/index.php/indicadores-del-ano

Dávila, M. (2012). Diseño de una plataforma de software para televisión digital interactiva de un canal de deportes utilizando Ginga-NCL LUA.

dos Santos, D., do Vale, D., \& Pedroso, L. (2006). Digital TV and Distance Learning: Potentials and Limitations. Frontiers in Education Conference, (pp. 1-6).
Educa. (s/f). From http://www.educa.ed

Fundación Ecuador. (2010). From http:// fe.org.ec/

Ierusalimschy, R., de Figueiredo, L., \& Celes, W. (2006). Lua 5.I Reference Manual.

Instituto Nacional de Estadísticas y Censos INEC. (s/f). From http://www.ecuadorencifras.gob.ed/

International Telecommunication Union. (2011). Nested context language and Ginga-NCL.

Jiménez, J., \& Moreno, G. (2012). CYCLE OF PDCA T-LEARING MODEL AND ITS APPLICATION ON INTERACTIVE DIGITAL TV. Scielo

Lamont, S. (2003). An 8-Step Process for Creating Compelling Enhanced Television. Procedings of the Euro iTV.

Ling-Lang, T., Che-Han, H., \& K., O. (2014). Acceptance of web-based e-learning systems: Behavioral intention and impacts.

Lytras, M., Lougos, C., P., C., \& P. A. (2002). Interactive Television and e-Leaming Convergence: Examining the Potential of t-Learning.

Ministerio de Telecomunicaciones y de la Sociedad de la Información. (s/f). From http://www.telecomunicaciones.gob.ec/ television-digital-terrestre-en-el-ecuador/

Nested Context Language. (s/f). From http://www.ncl.org.br/en/inicio

Nicolussi, R. (2005). Prospecttive formative del futuro: il t-learning.

Organización de Naciones Unidas ONU. (s/f). From http://www.un.org/es/roadsafety/background.shtml 
Pindado, J. (2006). T-LEARNING EL POTENCIAL EDUCATIVO DE LA TELEVISIÓN DIGITAL INTERACTIVA. Comunicar: revista científica iberoamericana de comunicación y educación, 93-101.

Pontificia Universidad Católica de Río de Janeiro. (s/f). From http://www.lua.org/

Reyes, A., \& Moreno, G. (2009). Aplicativo t-learning en la Televisión Digital Terrestre. International Journal of New Computer Architectures And Their Applications.

Reyes, A., Moreno, G., \& Rosero, C. (2010). Una aproximación a la Televisión digital y el T-learning. Latin American and Caribbean Conference for Engineering and Technology.
Sandoval, F. (2011). ISDB-T e ISDB-Tb: Integrated Service Digital Broadcasting Terrestrial. From Slideshare: http:// es.slideshare.net/blog_fralbe/7-isdb\#

Unicef. (s/f). Retrieved 16 de julio de 2013 from http://www.unicef.org/lac/media_18149.htm

Villanueva, J., \& Velásquez, C. (2010). Informe Preliminar: Estado del Arte de Receptores Set-Top-Box - Aplicaciones. Lima.

Zajc, M., Alič, K., Battelino, I., \& Tasič, J. (2007). Challenges of Interactive Digital Television for t-Learning. Zbornik Slovenske elektrotehniške konference ERK.

\section{Salvador Encalada Chérrez}

Ing. en Sistemas de la Universidad Espíritu Santo - Ecuador

E-mail: saencalada@uees.edu.ec

\section{Ivan Silva Feraud}

Ing. en Ciencias Computacionales

Máster Universitario en Ciencias Tecnológicas de la Computación

Docente tiempo completo de la Universidad Espiritu Santo-Ecuador

E-mail: ivansilva@gmail.com 